\title{
SINR Order Statistics in OFDMA Systems
}

\author{
Radha Krishna Ganti \\ Department of Electrical Engineering \\ Indian Institute of Technology, Madras \\ Chennai, India 600036 \\ rganti@ee.iitm.ac.in
}

\author{
Kiran Kuchi \\ Department of Electrical Engineering \\ Indian Institute of Technology, Hyderabad \\ Hyderabad, India 502205 \\ kkuchi@iith.ac.in
}

\begin{abstract}
In orthogonal frequency division multiple access downlink, the signal-to-interference-noise ratio (SINR) of every subband at each user is estimated with the aid of pilots and the channel quality (some function of SINR's) is fed back to the base station (BS). This information is utilized by the BS for downlink scheduling. However, the SINR of the bands at each user are correlated due to the common interfering BSs. In this paper, we obtain the order statistics of band SINR's by considering the the co-channel interference and taking the correlations of interference across the bands into consideration. The order statistics are then used to analyse a best- $m$ band feedback scheme, in which every users reports the index of their best $m$ bands to the BS. The BS utilizes this information and does greedy allocation of the bands to the users. We observe that utilizing the partial ordering of SINR's for channel allocation provides substantial gains over random allocation of bands to the users even in the presence of interference.
\end{abstract}

\section{INTRODUCTION}

Orthogonal frequency division multiple access (OFDMA) is the ubiquitous downlink access technique in the next generation cellular standards. In OFDMA, the users utilize the pilots to estimate the channel quality of a physical resource block which we term as "band" for convenience. The users then report the channel quality (or some functions of them, for e.g., exponential effective SNR mapping (EESM)) of the best $m$-bands to the BS, which uses this information for scheduling the users. Hence the order statistics of the SINR's of different bands are important in the design and the analysis of the downlink scheduler.

Interference from the other BSs critically influence the joint statistics of SINRs of the bands. At each user, while the small scale fading from the interfering BSs is independent across the bands, all the bands encounter the same path loss from the interfering BSs. Hence the interference is correlated across the bands.

In the earlier works, only the signal-to-noise ratio is considered and the interference from other BSs is generally neglected in the analysis of order statistics. The main contribution of the paper is obtaining the order statistics of SINR's of the bands by explicitly taking into account the interference and its correlations across the bands. We use a spatial Poisson point process [1], [2] for the BS locations. This spatial model was introduced in [3] to model the interference in a cellular network. From the order statistics, we observe that in wideband systems with high frequency selectivity (and hence a large number of bands) there is a substantial gain using the best band scheduling even with interference. The obtained order statistics can potentially be used to analyse various schedulers. As an application, we consider a simple band allocation scheme in which the users feedback the order of their best- $m$ bands to the BS, and the BS uses this information to greedily schedule the users.

The results closest to our work are presented in [4], [5]. The order statistics of SNR in an OFDMA system, and the best$m$ feedback scheme are analyzed, however the interference from other BSs is neglected. Most performance evaluation used simulations or resort to asymptotics [6], [7]. In [8] a single-bit feedback scheme for OFDMA is analyzed, while in [9] an approximation of the distribution of EESM is provided. In [10] asymptotics and bounds on sum rate are provided for an OFDMA system.

In Section II, we introduce the system model and assumptions. In Section III, we obtain the order statistics of the SINR bands and we use these results in IV to obtain the coverage probability for the best- $m$ band channel allocation.

\section{SySTEM MODEL}

The locations of the BSs are modeled by a spatial Poisson Poisson process $\Phi$ of density $\lambda$. See Figure 1 for a typical realization of the BS locations. The merits and demerits of this model are discussed in [3]. For notational simplicity we represent a node by its location. The path loss is given by $\ell(x)=\|x\|^{-\alpha}$ where $\alpha$ is the path loss exponent and we assume that $\alpha>2$.

A mobile user associates itself with the closest BS. So the region served by a BS corresponds to its Voronoi cell with respect to the other BSs. We consider an OFDMA based downlink. In OFDMA, the BS clubs resources either in time and frequency into resource blocks and allocates them to the users depending on the channel conditions. We assume that the resource blocks are clubbed into $L$ bands. With the aid of pilots, each user estimates the SINR in each band and feeds back some of this information to the BS, using which the BS allocates the bands to the users.

Since we are interested in the downlink performance, without loss of generality, we consider a typical mobile user at the origin denoted by $o$ and analyze the SINR distribution in different bands with respect to this user. The small-scale fading (power) between the typical mobile and an interfering base station $x \in \Phi$ in the $i$-th band is given by $\mathrm{h}_{x, i}$. We assume Rayleigh fading, which implies that $\mathrm{h}_{x, i}$ is exponentially distributed. We also set $\mathbb{E}\left[\mathrm{h}_{x, i}\right]=1$, and assume that the fading is independent across nodes and bands.

We denote the closest BS the typical user at $o$ by $z \in \Phi$. 


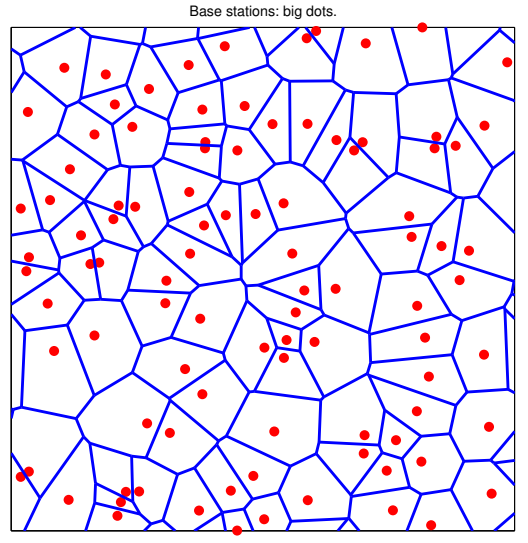

Fig. 1. A typical realization of the BS locations distributed as spatial Poisson point process and their associated cells.

The downlink SINR of the $i$-th band is given by

$$
\gamma_{i}=\frac{\mathrm{h}_{z, i}\|z\|^{-\alpha}}{\sigma^{2}+\sum_{x \in \Phi \backslash\{z\}} \mathrm{h}_{x, i}\|x\|^{-\alpha}},
$$

where $\sigma^{2}$ is the noise variance. We assume that all the BSs transmit with unit power. A node is set to be in coverage if its received SINR (in its scheduled band) is greater than a threshold $\theta$.

\section{ORDER STATISTICS OF SINR}

The order statistics of the $\gamma_{i}$ are necessary for computing the coverage probability of a user. It is easy to see that they are identical random variables (the expressions of $\gamma_{i}$ 's are similar). If $\gamma_{i}, i=1 \ldots L$, were independent, computing the ordered statistics is easy [11]. But the SINR's are not independent because of the common interferer locations $\Phi$ which introduces correlates across all the $\gamma_{i}$.

In this section we obtain the ordered distribution of the bands i.e., $\mathbb{P}\left(\gamma_{i} \leq \theta\right)$ given that $\gamma_{L} \geq \gamma_{L-1} \geq \ldots \geq \gamma_{1}$ also accounting for the correlations in $\gamma_{i}$. We first begin with the distribution of the best band $\gamma_{L}$ and we will extended this result to the other ordered bands in Lemma 1.

Theorem 1. The CDF of the maximum SINR band is given by

$$
\begin{aligned}
\mathcal{P}(L, \theta) & =\sum_{k=0}^{L}\left(\begin{array}{l}
L \\
k
\end{array}\right)(-1)^{k} . \\
& \int_{0}^{\infty} 2 \pi \lambda r e^{-k \theta r^{\alpha} \sigma^{2}-\pi \lambda r^{2} c(\alpha, k, \theta)} \mathrm{d} r,
\end{aligned}
$$

where

$$
c(\alpha, k, \theta)=2 F 1\left(k, \frac{-2}{\alpha}, 1-\frac{2}{\alpha},-\theta\right),
$$

and ${ }_{2} F_{1}(a, b, c, z)$ is the standard hypergeometric function ${ }^{1}$.

Proof: See Appendix A

$$
{ }_{2} F_{1}(a, b, c, z)=\frac{\Gamma(c)}{\Gamma(b) \Gamma(c-b)} \int_{0}^{1} \frac{t^{b-1}(1-t)^{c-b-1}}{(1-t z)^{a}} \mathrm{~d} t .
$$

The result in Theorem 1 can be simplified in some special cases. For example, in an interference-limited regime i.e., $\sigma^{2}=$ 0 , it is easy to see that the integral in (1) can be simplified to

$$
\mathcal{P}(L, \theta)=\sum_{k=0}^{L}\left(\begin{array}{l}
L \\
k
\end{array}\right) \frac{(-1)^{k}}{c(\alpha, k, \theta)} .
$$

Observe that the distribution does not depend on the density of the BSs. Also, for $\alpha=4$ it can be shown that

$$
\mathcal{P}(L, \theta)=1+\sum_{k=1}^{L}\left(\begin{array}{l}
L \\
k
\end{array}\right) \frac{(-1)^{k} \pi^{3 / 2} \lambda}{2 \sqrt{k \theta \sigma^{2}}} e^{\eta_{k}^{2}} \operatorname{erfc}\left(\eta_{k}\right),
$$

where $\eta_{k}=\frac{c(4, k, \theta) \pi \lambda}{2 \sqrt{k \theta \sigma^{2}}}$. In Figure 2, the $\operatorname{CDF} \mathcal{P}(L, \theta)$ is plotted

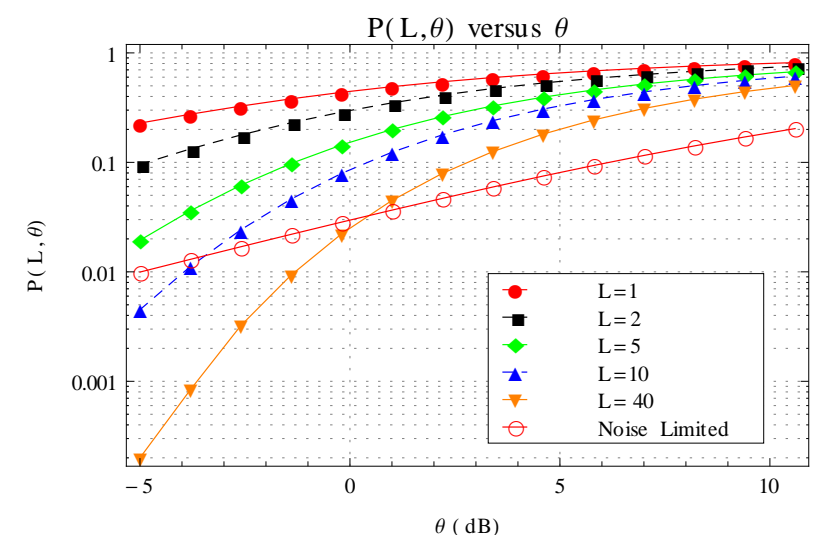

Fig. 2. The outage probability $\mathcal{P}(L, \theta)$ as a function of $\theta$ for different $L$ with $\alpha=4, \sigma^{2}=0.1$ and $\lambda=0.25$. The average distance of the nearest BS is $(2 \sqrt{\lambda})^{-1}$ and hence $\sigma^{2}=0.01$ correspond to an average receive signalto-noise ratio of $\left(16 \lambda^{2} \sigma^{2}\right)^{-1} \approx 20 \mathrm{~dB}$. The outage probability curve with no interference and a single band (denoted as "Noise Limited") is also shown.

as a function of $\theta$ for different $L$. As expected, when the number of bands increase, the outage probability for the best band decreases. Also observe the diminishing returns in using 40 bands over 10 bands. For example with $\theta=1(0 \mathrm{~dB})$ SINR threshold, there is a $80 \%$ reduction in the outage when using 20 bands compared to an additional $10 \%$ reduction by using 30 extra bands. We now compute the distribution of all the other ordered bands.

Lemma 1. Let $\gamma_{L} \geq \gamma_{L-1} \geq \ldots \geq \gamma_{1}$, then the probability that $\mathbb{P}\left(\gamma_{n} \leq \theta\right)$ which we denote by $\mathcal{P}(n, \theta)$ is given by

$$
\begin{aligned}
\mathcal{P}(n, \theta)= & \sum_{k=n}^{L}\left(\begin{array}{l}
L \\
k
\end{array}\right) \sum_{j=0}^{k}\left(\begin{array}{l}
k \\
j
\end{array}\right)(-1)^{j} \\
& \cdot \int_{0}^{\infty} 2 \pi \lambda r e^{-(L-k+j) \theta r^{\alpha} \sigma^{2}} e^{-\pi \lambda r^{2} c(\alpha, L-k+j, \theta)} \mathrm{d} r .
\end{aligned}
$$

Proof: From the proof of Theorem 1, the SINR's are independent and identical conditioned on the underlying node locations $\Phi$. Denote the common CDF of the SINR conditioned on $\Phi$ as $F(x)$. Then the ordered statistics [11] equal

$$
\mathbb{P}\left(\gamma_{n} \leq \theta \mid \Phi\right)=\sum_{k=n}^{L}\left(\begin{array}{l}
L \\
k
\end{array}\right) F(\theta)^{k}(1-F(\theta))^{L-k} .
$$


As in the proof of Theorem (1) we set $F(\theta)=1-g(\Phi, \theta)$. Averaging over $\Phi$, we obtain

$$
\mathbb{P}\left(\gamma_{n} \leq \theta\right)=\sum_{k=m}^{L}\left(\begin{array}{l}
L \\
k
\end{array}\right) \mathbb{E}\left[(1-g(\Phi, \theta))^{k} g(\Phi, \theta)^{L-k}\right],
$$

which after binomial expansion equals,

$$
\mathbb{P}\left(\gamma_{n} \leq \theta\right)=\sum_{k=m}^{L}\left(\begin{array}{l}
L \\
k
\end{array}\right) \sum_{j=0}^{k}\left(\begin{array}{l}
k \\
j
\end{array}\right)(-1)^{j} \mathbb{E}\left[g(\Phi, \theta)^{L-k+j}\right] .
$$

Using (3) and substituting for $\mathbb{E}\left[g(\Phi, \theta)^{L-k+j}\right]$, we obtain the result.

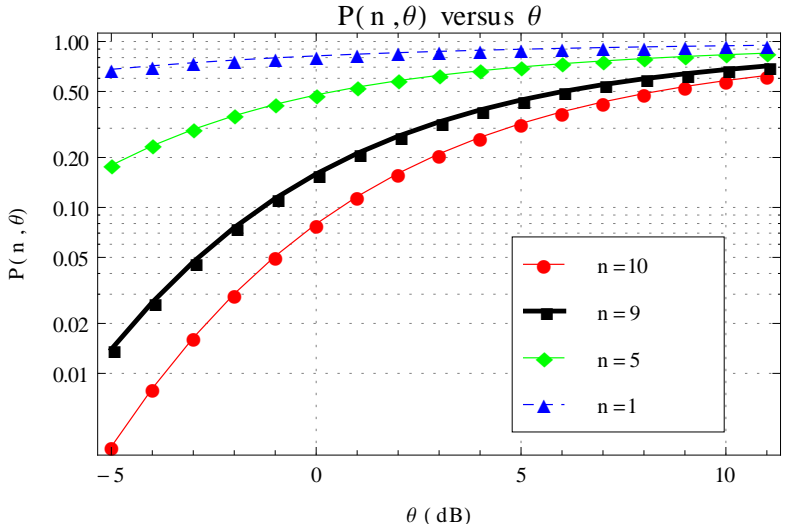

Fig. 3. The $\operatorname{CDF} \mathcal{P}(n, \theta)$ as a function of $\theta$ for different $n$ with $L=10$ bands, $\sigma^{2}=0$ and $\alpha=4$.

As before, the above expression can be further simplified in an interference limited regime or $\sigma^{2}=0$ and equals,

$$
\mathcal{P}(n, \theta)=\sum_{k=n}^{L}\left(\begin{array}{l}
L \\
k
\end{array}\right) \sum_{j=0}^{k}\left(\begin{array}{l}
k \\
j
\end{array}\right) \frac{(-1)^{j}}{c(\alpha, L-k+j, \theta)} .
$$

In Figure 3, the CDF's of the different bands are plotted, from which we can immediately infer the gain in using the best band over the worst band. All the joint distributions of the ordered $\gamma_{i}$ 's can be obtained using the techniques presented in the proofs of Theorem 1 and Lemma 1. In the next lemma we quantify the SINR gain in using the best band over the worst band. The gain is defined as $G=\gamma_{L} / \gamma_{1}$. This metric quantifies the advantage of scheduling the best band over a random allocation. For simplicity, we just consider the nonoise case. We now derive the CDF of $G$.

Lemma 2. When $\sigma^{2}=0$, the distribution of the ratio of the SINR of the best band to that of the SINR of the worst band is given by

$$
\begin{aligned}
& \mathbb{P}(G \leq t)=L \sum_{k=0}^{L-1}\left(\begin{array}{c}
L-1 \\
k
\end{array}\right) \frac{2(-1)^{L-1-k}}{\alpha-2} . \\
& \int_{0}^{\infty} \frac{\mathrm{AF}_{1}\left(1-\frac{2}{\alpha}, L-k+1, k, 2-\frac{2}{\alpha},-a, \frac{-a}{t}\right)}{\mathrm{AF}_{1}\left(-\frac{2}{\alpha}, L-k, k, 1-\frac{2}{\alpha},-a, \frac{-a}{t}\right)^{2}} \mathrm{~d} a
\end{aligned}
$$

for $t>1$. In the above expression, $\mathrm{AF}_{1}$ denote the Appell hypergeometric function ${ }^{2}$.

Proof: See Appendix B.

In Figure 4, the CDF of the gain $G$ is plotted. We observe that with high probability the gain is greater than 10 . In the

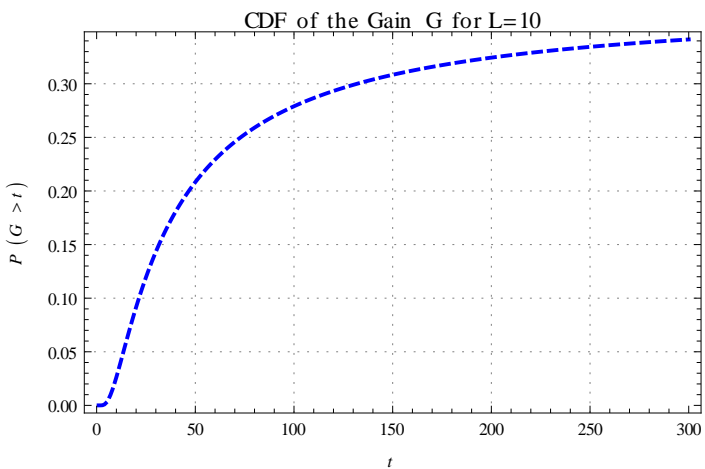

Fig. 4. The CDF of the gain $G$ for $L=10$ bands, $\sigma^{2}=0$ and $\alpha=4$.

next section we utilize the order statistics developed in this section to analyze a simple channel allocation policy of which the best band scheduling is a special case. While we focus on coverage, other metrics like ergodic rate can also be computed using the order statistics.

\section{BEST- $m$ CHANNEL ALLOCATION POLICY}

Using pilots, every mobile user will be able to estimate the SINR in each band. So a mobile user can order the quality of his bands based on the computed SINR and report the order to the BS. We assume each mobile user reports the order of $m$ best bands. In addition, each user usually reports an exponential effective SNR mapping (EESM) value (a scalar) that can be used by the BS to estimate the "quality" of the bands [9]. This information is required for rate allocation. However, in the current paper we do not consider this information and is a subject of future work.

In this section for simplicity, we assume that the number of users being served by a BS is equal to the number of bands $L$. The BS receives this band ordering from all its users and allocates the bands in the following greedy manner.

1) The BS first considers the best bands of all the users. For each band $b_{i}, i=1, \ldots, L$, the BS lists all the users who reported $b_{i}$ as their best band. If only a single user reported $b_{i}$ as its best band, it allocates the band to that user. If multiple users reported $b_{i}$ as their best band, the $\mathrm{BS}$ randomly picks one of these users and allocates the band to that user.

2) The BS then considers only the unallocated bands and the second best bands of the remaining users (users whose best band was not allocated) and repeats the above procedure with respect to the second best band.

${ }^{2} \mathrm{AF}_{1}\left(\alpha, \beta, \beta^{\prime}, \gamma, x, y\right)=\frac{\Gamma(\gamma)}{\Gamma(\alpha) \Gamma(\gamma-\alpha)} \int_{0}^{1} u^{\alpha-1}(1-u)^{\gamma-\alpha-1}(1-$ $u x)^{-\beta}(1-u y)^{-\beta^{\prime}} \mathrm{d} u$. Evaluated in Mathematica as AppellF1. 
3) This allocation continues till $m$-times with the BS focusing on the $i$-th best bands of the users in the $i$-th time.

For illustration, let $L=m=3$, i.e., there are 3 bands, 3 users, and the users report their entire band order. Suppose $\{1,2,3\}$, $\{3,2,1\},\{3,2,1\}$ are the reported band orders of the three users $u_{1}, u_{2}, u_{3}$. The BS first considers the best bands of the three users which are 1 for $u_{1}, 3$ for $u_{2}$ and 3 for $u_{3}$. Hence the BS allocates frequency 1 to $u_{1}$. Since both users $u_{2}$ and $u_{3}$ reported band 3 as their best band, it randomly picks one of the user to allocate band 3. Let us suppose the band 3 is allocated to user 3 . Observe that band 2 is not yet allocated to any user. The BS now considers the second best band of the remaining users which is $u_{2}$. Its reported second best band is 1 , which was allocated to user $u_{1}$. So the BS now considers the third best band and the remaining users. For the user $u_{2}$ the third best band is 2 which was not allocated earlier. So the BS allocates the band 2 to $u_{2}$.

From the allocation algorithm, it is easy to see that if $m<$ $L$, then some of the users might not be allocated any frequency bands and also some of the frequency bands might not be allocated to any user. However, if the number of reported bands $m=L$, then every users are allocated some frequency band. The following lemmas show that the band ordering of various users is independent, a fact we will be using to analyse the allocation probabilities.

Lemma 3. For any user, any order of the bands is equally likely, i.e., the SINR order of the bands is equally likey to be any one of the permutations of $\{1, \ldots, L\}$.

Proof: The result is obvious when conditioned on the location of the BSs $\Phi$, since the fading coefficients are independent. Hence

$$
\mathrm{P}\left(\text { Order }=\left(i_{1}, \ldots, i_{L}\right) \mid \phi\right)=\frac{1}{L !} .
$$

Averaging over $\phi$ we get the result.

Lemma 4. The band ordering across users is independent.

Proof: Let $O_{1}$ denote the band order of user 1 and $\mathrm{O}_{2}$ the band order of user 2. Since the fading variables are independent across nodes and bands, conditioned on the point process we have

$$
\begin{aligned}
& \mathbb{P}\left(O_{1}=\left(i_{1}, \ldots, i_{L}\right), O_{2}=\left(j_{1}, \ldots j_{L}\right) \mid \phi\right) \\
& =\mathbb{P}\left(O_{1}=\left(i_{1}, \ldots, i_{L}\right) \mid \phi\right) \mathbb{P}\left(O_{2}=\left(j_{1}, \ldots j_{L}\right) \mid \phi\right) \stackrel{(a)}{=} \frac{1}{L !} \frac{1}{L !},
\end{aligned}
$$

where $(a)$ follows from Lemma 3. Averaging over $\phi$ we obtain the result.

Consider a typical user (randomly picked user from the $L$ users) $u$ and let $\mathrm{P}_{i}$ denote the probability that the user is allocated his $i$-th best band. So for $m<L, \sum_{i=1}^{m} \mathrm{P}_{i}<1$, and the probability that a user is not scheduled is $1-\sum_{i=1}^{m} \mathrm{P}_{i}$. However, $\sum_{i=1}^{L} \mathrm{P}_{i}=1$.

From the allocation policy, it can be easily seen that evaluation of $\mathrm{P}_{i}$ is purely combinatorial. In fact $\mathrm{P}_{i}$ is a function of only $L$ and $m$ and does not depend on the actual SINR values, but just the band ordering of the users. However from Lemma 3 and 4, we observe that for a user any ordering is equally likely and the ordering is independent across users. The allocation strategy is very similar to that of the Balls and Urns problem which has been studied extensively. For example, let us consider the first step in the allocation, i.e., the BS allocating the best band. Consider the $L$ frequency bands as $L$ urns. The best band of each user is equally likely and independent of the other users. Hence we can think of each user placing a ball (with his number/id) in one of the urn independently and uniformly. So the probability that a typical user $u$ is allocated his best band can be computed by analysing the simple balls and urns problem, and from basic combinatorics can be computed to be

$$
\mathrm{P}_{1}=\sum_{a=0}^{L-1} \frac{1}{1+a}\left(\begin{array}{c}
L-1 \\
a
\end{array}\right)\left(\frac{1}{L}\right)^{l}\left(1-\frac{1}{L}\right)^{L-1-a},
$$

where $1 /(1+a)$ is the probability of the typical user $u$ being selected given that there are $a$ users in one bin (over and above the particular user in consideration). The remaining terms are the probability of there being $a$ users. The above expression can be simplified to $\mathrm{P}_{1}=1-\left(1-\frac{1}{L}\right)^{L}$. So as the number of users/bands $L$ increase to infinity, the probability that a user is allocated his best band approaches $1-e^{-1} \approx 0.63$. Similarly, the probability that the user is allocated $i$-th best band can be computed by looking at the distribution of the empty bins [12]. Also, for $m<L$ the probability mass function (PMF) of the number of unallocated bands can be computed. However, this is a tedious recursive exercise in combinatorial probability that leads to large expressions that offer no additional insight. Instead we obtain $\mathrm{P}_{1}, \ldots \mathrm{P}_{L}$ using simulations. See Figure 5 for the distribution when $L=10$. Observe that the obtained distribution works for any $m \leq L$ by just considering the first $m$ terms of the distribution.

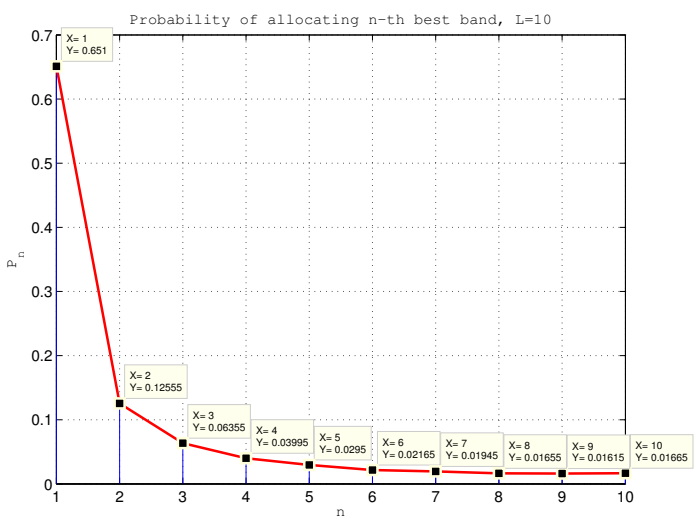

Fig. 5. PMF $\mathrm{P}_{i}, i=1, \ldots, 10$ for $L=10$ bands. Observe that with a high probability a user will be allocated one of his two best bands. Also $\mathrm{P}_{1}=0.65$ which is very close to 0.63 the $L \rightarrow \infty$ limit.

\section{A. Coverage Probability}

The coverage probability of an user depends on the band that is provided by the BS using the allocation scheme described in the previous section. If the user is allocated his 
best channel, then the coverage probability is $\mathbb{P}\left(\gamma_{L} \geq \theta\right)$ (recall that $\gamma_{L} \geq \gamma_{L-1} \geq \ldots \geq \gamma_{1}$ are the ordered SINR values). Instead if the user is allocated his second best channel, then the coverage probability is $\mathbb{P}\left(\gamma_{L-1} \geq \theta\right)$. Hence is easy to observe that the probability of coverage for a typical is $\mathrm{P}_{c}=\sum_{i=1}^{m} \mathbb{P}\left(\gamma_{L-i+1} \geq \theta\right) \mathrm{P}_{i}$, which equals

$$
\mathrm{P}_{c}=\sum_{i=1}^{m}(1-\mathcal{P}(L-i+1, \theta)) \mathrm{P}_{i} .
$$

For example, for $L=10$ and $m=2$, from Figure 5 we have $\mathrm{P}_{c} \approx 0.65 \mathbb{P}\left(\gamma_{L} \geq \theta\right)+0.126 \mathbb{P}\left(\gamma_{L-1} \geq \theta\right)$. The probabilities $\mathcal{P}(L-i+1, \theta)$ are provided by Lemma 1 .

\section{B. Comparison with random frequency allocation}

We will now evaluate the outage probability when the BS randomly allocated a frequency band to each user instead of trying to allocate the best band. In this case, it is easy to see that the coverage probability follows from Theorem 1, by setting $L=1$. This is because, we can consider a typical user and allocate him some random band, which amounts to setting the number of bands to 1 . So

$$
\mathrm{P}_{r}=\int_{0}^{\infty} 2 \pi \lambda r e^{-\theta r^{\alpha} \sigma^{2}} e^{-\pi \lambda r^{2} c(\alpha, 1, \theta)} \mathrm{d} r
$$

which without noise equals $c(\alpha, 1, \theta)^{-1}$. This result equals the coverage result presented in [3]. In Figure 6, the coverage probability is plotted as a function of the SINR threshold $\theta$ for different $m$. Also the coverage probability with random allocation $\mathrm{P}_{r}$ is plotted. We observe that even 2-best band feedback increases the coverage over a random allocation. Also observe the diminishing returns after $m>5$ which is more evident from Figure 7. Hence even a little feedback in terms of band ordering can provide great improvements in coverage probability.

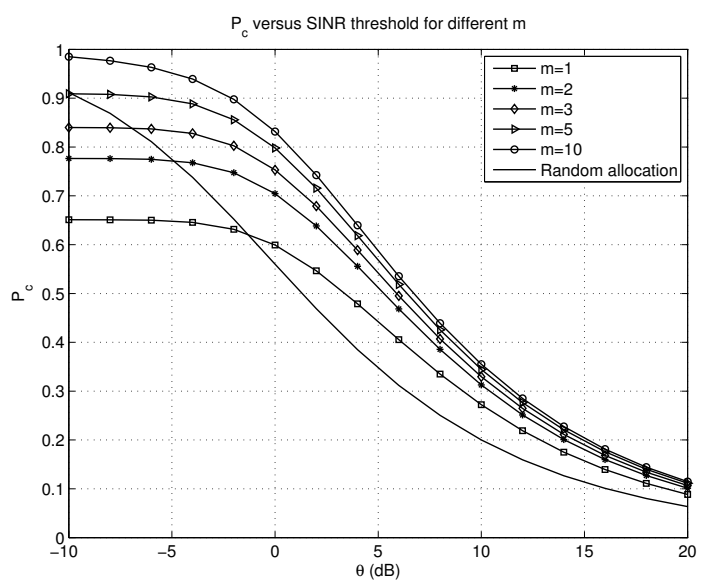

Fig. 6. Coverage probability $\mathrm{P}_{c}$ versus the SINR threshold $\theta$ for the no-noise case $\sigma^{2}=0$ and $\alpha=4$. The multiple curves correspond to different values of $m$, i.e., the number of best bands that a user feeds back. Also the coverage probability $\mathrm{P}_{r}$ with random allocation is plotted.

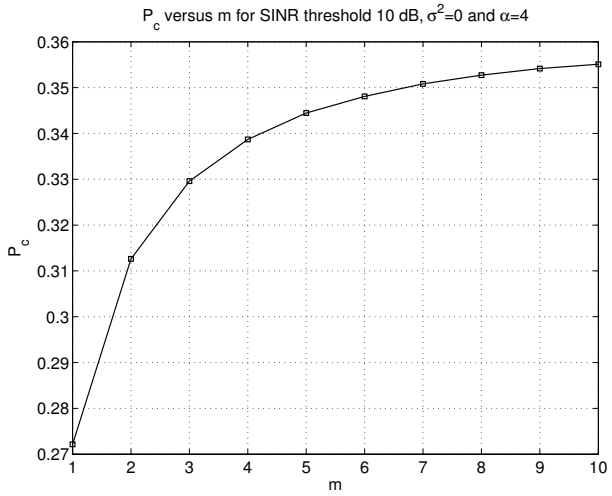

Fig. 7. Coverage probability $\mathrm{P}_{c}$ versus the feedback $m$ for SINR threshold of 10 -dB for the no-noise case $\sigma^{2}=0$ and $\alpha=4$. Observe the diminishing returns as $m$ increases.

\section{REFERENCES}

[1] D. Stoyan, W. S. Kendall, and J. Mecke, Stochastic Geometry and its Applications, 2nd ed., ser. Wiley series in probability and mathematical statistics. New York: Wiley, 1995.

[2] J. Kingman, Poisson Process. Oxford University Press, 1993.

[3] J. G. Andrews, F. Baccelli, and R. K. Ganti, "A tractable approach to coverage and rate in cellular networks," IEEE Trans. on Communications, vol. 59, pp. 3122 - 3134, Nov. 2011. [Online]. Available: http://arxiv.org/abs/1009.0516

[4] J. Leinonen, J. Hamalainen, and M. Juntti, "Performance analysis of downlink OFDMA resource allocation with limited feedback," IEEE Trans. on Wireless Communications, vol. 8, no. 6, pp. 2927 -2937, Jun. 2009.

[5] P. Viswanath, D. Tse, and R. Laroia, "Opportunistic beamforming using dumb antennas," IEEE Trans. on Info. Theory, vol. 48, no. 6, pp. 12771294, 2002.

[6] J. Chen, R. Berry, and M. Honig, "Large system performance of downlink OFDMA with limited feedback," in Proc., IEEE Intl. Symposium on Information Theory, Jul. 2006, pp. $1399-1403$.

[7] R. Kwan, C. Leung, and J. Zhang, "Proportional fair multiuser scheduling in lte," Signal Processing Letters, IEEE, vol. 16, no. 6, pp. $461-464$, Jun. 2009.

[8] S. Sanayei and A. Nosratinia, "Opportunistic downlink transmission with limited feedback," IEEE Trans. on Info. Theory, vol. 53, no. 11, pp. 4363 -4372 , Nov. 2007.

[9] S. Donthi and N. Mehta, "An accurate model for EESM and its application to analysis of CQI feedback schemes and scheduling in LTE," IEEE Trans. on Wireless Communications, vol. 10, no. 10, pp. $3436-3448$, Oct. 2011

[10] R. Aggarwal, C. E. Koksal, and P. Schniter, "Scaling laws and design principles for multi-cellular wireless OFDMA systems," CoRR, vol. abs/1108.3780, 2011.

[11] H. David and H. Nagaraja, Order statistics. Wiley Online Library, 1970.

[12] V. Kolchin, B. Sevastianov, and V. Chistiakov, Random allocations. Vh Winston, 1978.

\section{APPENDIX A \\ PROOF OF THEOREM 1}

Proof: The outage probability of the typical node is given

by

$$
\mathcal{P}(L, \theta)=\mathbb{P}\left(\max \left\{\gamma_{1}, \ldots, \gamma_{L}\right\} \leq \theta\right) .
$$

which implies

$$
\begin{aligned}
\mathcal{P}(L, \theta)= & \mathbb{P}\left(\frac{\mathrm{h}_{z, 1}\|z\|^{-\alpha}}{\sigma^{2}+\sum_{x \in \Phi \backslash\{z\}} \mathrm{h}_{x, 1}\|x\|^{-\alpha}} \leq \theta, \ldots,\right. \\
& \left.\frac{\mathrm{h}_{z, L}\|z\|^{-\alpha}}{\sigma^{2}+\sum_{x \in \Phi \backslash\{z\}} \mathrm{h}_{x, L}\|x\|^{-\alpha}} \leq \theta\right)
\end{aligned}
$$


Since $\mathrm{h}_{z, i}$ the fades across different bands to the desirable BS are independent, we have

$$
\mathcal{P}(L, \theta)=\mathbb{E} \prod_{k=1}^{L} 1-e^{-\theta\|z\|^{\alpha}\left(\sigma^{2}+\sum_{x \in \Phi \backslash\{z\}} \mathrm{h}_{x, k}\|x\|^{-\alpha}\right)},
$$

where the expectation is with respect to all the other random variables, i.e., $z, \Phi$ and the fades of the interfering BSs. Converting the exponent of sums into product of exponents, we obtain

$\mathcal{P}(L, \theta)=\mathbb{E}\left[\prod_{k=1}^{L} 1-e^{-\theta\|z\|^{\alpha} \sigma^{2}} \prod_{x \in \Phi \backslash\{z\}} e^{-\theta\|z\|^{\alpha} \mathrm{h}_{x, k}\|x\|^{-\alpha}}\right]$.

Sine $\mathrm{h}_{x, k}$ are independent for different $k$ and $x$, the expectation with respect to these variables can be moved inside as

$$
\mathbb{E}\left[\prod_{k=1}^{L} 1-e^{-\theta\|z\|^{\alpha} \sigma^{2}} \prod_{x \in \Phi \backslash\{z\}} \mathbb{E}_{\mathrm{h}_{x, k}}\left[e^{-\theta\|z\|^{\alpha} \mathrm{h}_{x, k}\|x\|^{-\alpha}}\right]\right] .
$$

From the Laplace transform of an exponential random variable, it follows that $\mathcal{P}(L, \theta)=\mathbb{E} \prod_{k=1}^{L} 1-g(\Phi, \theta)$, where

$$
g(\Phi, \theta)=e^{-\theta\|z\|^{\alpha} \sigma^{2}} \prod_{x \in \Phi \backslash\{z\}} \frac{1}{1+\theta\|z\|^{\alpha}\|x\|^{-\alpha}}
$$

Using the binomial expansion and moving the expectation inside the summation,

$$
\mathcal{P}(L, \theta)=1+\sum_{k=1}^{L}\left(\begin{array}{l}
L \\
k
\end{array}\right)(-1)^{k} \mathbb{E} g(\Phi, \theta)^{k}
$$

We now evaluate $\mathbb{E}\left[g(\Phi, \theta)^{k}\right]$, by first evaluating $\mathbb{E}\left[\prod_{x \in \Phi \backslash\{z\}}\left(1+\theta\|z\|^{\alpha}\|x\|^{-\alpha}\right)^{-k}\right]$. Using the probability generating functional of the Poisson point process [1] and the strong Markov property of the PPP, the above expectation is

$$
\exp \left(-\lambda 2 \pi \int_{\|z\|}^{\infty}\left(1-\frac{1}{\left(1+\theta\|z\|^{\alpha} x^{-\alpha}\right)^{k}}\right) x \mathrm{~d} x\right),
$$

which after further simplification equals,

$$
\exp \left(-\lambda 2 \pi \theta^{2 / \alpha}\|z\|^{2} \int_{\theta^{-1 / \alpha}}^{\infty}\left(1-\frac{1}{\left(1+x^{-\alpha}\right)^{k}}\right) x \mathrm{~d} x\right) .
$$

The inner integral can be further simplified as

$$
\exp \left(-\pi \lambda\|z\|^{2}(-1+c(\alpha, k, \theta))\right.
$$

where

$$
c(\alpha, k, \theta)=2 F 1\left(k, \frac{-2}{\alpha}, 1-\frac{2}{\alpha},-\theta\right) .
$$

Hence

$\mathbb{E}\left[g(\Phi, \theta)^{k}\right]=\exp \left(-k \theta\|z\|^{\alpha} \sigma^{2}-\pi \lambda\|z\|^{2}(-1+c(\alpha, k, \theta))\right.$.

Using the distribution of the nearest neighbour distance in a PPP we now average out the distance $\|z\|$ which is given by $f(r)=2 \pi r \lambda \exp \left(-\lambda \pi r^{2}\right)$ [1], we obtain the result.
APPENDIX B

PROOF OF LEMMA 2

Proof: We have

$$
\mathbb{P}(G \leq t)=\mathbb{E}[\mathbb{P}(G \leq t \mid \Phi)]
$$

We first consider the conditional probability $\mathbb{P}(G \leq t \mid \Phi)$, and finally average over the location of nodes as in the previous lemma. From the theory of ordered random variables [11] and with basic algebra it follow that,

$$
\mathbb{P}(G \leq t \mid \Phi)=L \int_{o}^{\infty}[F(a / t)-F(a)]^{L-1} f(a) \mathrm{d} a,
$$

where $F(a)=1-g(\Phi, a)$ and $f(a)$ is the derivative of $F(a)$. For notational simplicity let $\hat{\Phi}=\Phi \backslash\{z\}$. Using the chain rule and (2), the derivative $f(a)$ can be computed to be,

$$
f(a)=\sum_{y \in \hat{\Phi}}\|z\|^{\alpha}\|y\|^{-\alpha} \eta_{z}(y, a)^{2} g(\hat{\Phi} \backslash\{y\}, a),
$$

where $\eta_{z}(x, a)=\left(1+a\|z\|^{\alpha}\|x\|^{-\alpha}\right)^{-1}$. Using binomial expansion and averaging over the point process we obtain,

$$
\begin{gathered}
P(G \leq t)=L \sum_{k=0}^{L-1}\left(\begin{array}{c}
L-1 \\
k
\end{array}\right)(-1)^{L-1-k} . \\
\int_{0}^{\infty} \mathbb{E}\left[g(\Phi, a / t)^{k} g(\Phi, a)^{L-1-k} f(a)\right] \mathrm{d} a .
\end{gathered}
$$

We now evaluate the inner expectation. Observe that $g(\Phi, a)=$ $\eta_{z}(y, a) g(\Phi \backslash\{y\}, a)$. The expectation equals

$$
\begin{aligned}
& E\left[\sum_{y \in \hat{\Phi}}\|z\|^{\alpha}\|y\|^{-\alpha} \eta_{z}(y, a)^{L-k+1} \eta_{z}(y, a / t)^{k} .\right. \\
& \left.g(\hat{\Phi} \backslash\{y\}, a)^{L-k} g(\hat{\Phi} \backslash\{y\}, a / t)^{k}\right] .
\end{aligned}
$$

Using Cambells Mecke theorem [1], the above expectation can be expressed as $\delta(z, a) \mathbb{E}\left[g(\hat{\Phi}, a)^{L-k} g(\hat{\Phi}, a / t)^{k}\right]$, where

$$
\delta(z, a)=2 \pi \lambda \int_{\|z\|}^{\infty}\|z\|^{\alpha} y^{-\alpha+1} \eta_{z}(y, a)^{L-k+1} \eta_{z}(y, a / t)^{k} \mathrm{~d} y
$$

which can be further simplified to

$$
\frac{2 \pi \lambda\|z\|^{2}}{\alpha-2} \mathrm{AF}_{1}\left(1-\frac{2}{\alpha}, L-k+1, k, 2-\frac{2}{\alpha},-a, \frac{-a}{t}\right) .
$$

As in the above lemma, $\mathbb{E}\left[g(\hat{\Phi}, a)^{L-k} g(\hat{\Phi}, a / t)^{k}\right]$ equals

$$
e^{-\lambda 2 \pi\|z\|^{2} \int_{1}^{\infty}\left(1-\eta_{1}(x, a)^{L-k} \eta_{1}(x, a / t)^{k}\right) x \mathrm{~d} x} .
$$

The inner integral can be further simplified to $\frac{1}{2}\left(-1+\mathrm{AF}_{1}\left(-\frac{2}{\alpha}, L-k, k, 1-\frac{2}{\alpha},-a, \frac{-a}{t}\right)\right)$. Combining everything and averaging out the distance $\|z\|$, we obtain

$$
\begin{aligned}
& \mathbb{E}\left[g(\Phi, a / t)^{k} g(\Phi, a)^{L-1-k} f(a)\right]= \\
& \frac{2}{\alpha-2} \frac{\mathrm{AF}_{1}\left(1-\frac{2}{\alpha}, L-k+1, k, 2-\frac{2}{\alpha},-a, \frac{-a}{t}\right)}{\mathrm{AF}_{1}\left(-\frac{2}{\alpha}, L-k, k, 1-\frac{2}{\alpha},-a, \frac{-a}{t}\right)^{2}} .
\end{aligned}
$$

